\title{
Derangement polynomials with a complex variable
}

\author{
Abdelkader Benyattou \\ Department of Mathematics and Informatics, University of Djelfa, Algeria \\ RECITS Laboratory, P. O. 32 Box 32, El Alia 16111, Algiers, Algeria \\ e-mail: abdelkaderbenyattou@gmail.com, a.benyattou@univ-djelfa.dz
}

Received: 13 June 2020

Revised: 20 October 2020

Accepted: 2 November 2020

Abstract: In this paper, we define new polynomials with a complex variable related to the derangement polynomials and we give some properties of those polynomials. We use umbral calculus to establish a new congruence concerning the derangement polynomials with a complex variable.

Keywords: Derangement polynomials, Complex variable, Congruence, Umbral calculus.

2010 Mathematics Subject Classification: 11B83, 11A07, 30C10.

\section{Introduction}

Polynomials with a complex variable have attracted researchers' great interest, as the application of those polynomials appear in various fields of mathematics. The polynomials with a complex variable have been studied by various researchers for example, see $[3,6]$.

Derangement polynomials are defined by

$$
\mathcal{D}_{n}(x)=n ! \sum_{k=0}^{n} \frac{(x-1)^{k}}{k !} .
$$

It is clear that $\mathcal{D}_{n}(0)$ is the $n$-th derangement number, denoted by $\mathcal{D}_{n}$ counting the number of permutation of the set $[n]$ without a fixed point. The exponential generating function for the derangement polynomials is

$$
\sum_{n=0}^{\infty} \mathcal{D}_{n}(x) \frac{t^{n}}{n !}=\frac{e^{-t}}{1-t} e^{x t} .
$$

For more information about these numbers and polynomials one can see [7-9]. 
If we replace $x$ by $z$ or $\bar{z}$ in (1), where

$$
z=x+i y, \bar{z}=x-i y, i^{2}=-1,
$$

we get

$$
\begin{aligned}
& \sum_{n=0}^{\infty} \mathcal{D}_{n}(z) \frac{t^{n}}{n !}=\frac{e^{-t}}{1-t} e^{(x+i y) t}=\frac{e^{-t}}{1-t} e^{x t}(\cos (y t)+i \sin (y t)) \\
& \sum_{n=0}^{\infty} \mathcal{D}_{n}(\bar{z}) \frac{t^{n}}{n !}=\frac{e^{-t}}{1-t} e^{(x-i y) t}=\frac{e^{-t}}{1-t} e^{x t}(\cos (y t)-i \sin (y t)) .
\end{aligned}
$$

If we add or subtract the identities presented above, we get

$$
\begin{aligned}
& \sum_{n=0}^{\infty}\left[\mathcal{D}_{n}(z)+\mathcal{D}_{n}(\bar{z})\right] \frac{t^{n}}{n !}=\frac{2 e^{-t}}{1-t} e^{x t} \cos (y t) \\
& \sum_{n=0}^{\infty}\left[\mathcal{D}_{n}(z)-\mathcal{D}_{n}(\bar{z})\right] \frac{t^{n}}{n !}=\frac{2 i e^{-t}}{1-t} e^{x t} \sin (y t) .
\end{aligned}
$$

Let $\mathcal{D}_{n, 1}(z)=\mathcal{D}_{n}(z)+\mathcal{D}_{n}(\bar{z})$, and $\mathcal{D}_{n, 2}(z)=\mathcal{D}_{n}(z)-\mathcal{D}_{n}(\bar{z})$, then we have

$$
\begin{aligned}
\sum_{n=0}^{\infty} \mathcal{D}_{n, 1}(z) \frac{t^{n}}{n !} & =\frac{2 e^{-t}}{1-t} e^{x t} \cos (y t) \\
\sum_{n=0}^{\infty} \mathcal{D}_{n, 2}(z) \frac{t^{n}}{n !} & =\frac{2 i e^{-t}}{1-t} e^{x t} \sin (y t)
\end{aligned}
$$

and

$$
\begin{aligned}
\sum_{n=0}^{\infty} \mathcal{D}_{n}(z) \frac{t^{n}}{n !} & =\frac{e^{(-1+i y) t}}{1-t} e^{x t} \\
\sum_{n=0}^{\infty} \mathcal{D}_{n}(\bar{z}) \frac{t^{n}}{n !} & =\frac{e^{(-1-i y) t}}{1-t} e^{x t}
\end{aligned}
$$

That is now

$$
\cos (y t)=\frac{e^{i y t}+e^{-i y t}}{2}, \quad \sin (y t)=\frac{e^{i y t}-e^{-i y t}}{2 i},
$$

then

$$
\begin{aligned}
\sum_{n=0}^{\infty} \mathcal{D}_{n, 1}(z) \frac{t^{n}}{n !} & =\frac{e^{-t}}{1-t} e^{x t}\left(e^{i y t}+e^{-i y t}\right) \\
& =\sum_{n=0}^{\infty} \mathcal{D}_{n}(x) \frac{t^{n}}{n !} \sum_{n=0}^{\infty} \frac{\left[(i y t)^{n}+(-i y t)^{n}\right]}{n !} \\
& =\sum_{n=0}^{\infty} \mathcal{D}_{n}(x) \frac{t^{n}}{n !} \sum_{n=0}^{\infty}(i y)^{n}\left(1+(-1)^{n}\right) \frac{t^{n}}{n !} \\
& =\sum_{n=0}^{\infty} \frac{1}{n !} \sum_{k=0}^{n}\left(\begin{array}{l}
n \\
k
\end{array}\right) \mathcal{D}_{k}(x)(i y)^{n-k}\left(1+(-1)^{n-k}\right) t^{n} .
\end{aligned}
$$


Hence

$$
\begin{aligned}
& \mathcal{D}_{n, 1}(z)=\sum_{k=0}^{n}\left(\begin{array}{l}
n \\
k
\end{array}\right) \mathcal{D}_{k}(x)(i y)^{n-k}\left(1+(-1)^{n-k}\right), \\
& \mathcal{D}_{n, 2}(z)=\sum_{k=0}^{n}\left(\begin{array}{l}
n \\
k
\end{array}\right) \mathcal{D}_{k}(x)(i y)^{n-k}\left(1-(-1)^{n-k}\right) .
\end{aligned}
$$

The derangement polynomials with a complex variable can be defined by

$$
\mathcal{D}_{n}(z)=\sum_{k=0}^{n}\left(\begin{array}{l}
n \\
k
\end{array}\right) \mathcal{D}_{k}(x)(i y)^{n-k}
$$

and we can write $\mathcal{D}_{n}(z)$ as follows

$$
\mathcal{D}_{n}(z)=i^{n} \sum_{s=0}^{n}(-1)^{s}\left(\begin{array}{c}
n \\
2 s
\end{array}\right) \mathcal{D}_{2 s}(x) y^{n-2 s}-i^{n+1} \sum_{s=0}^{n}(-1)^{s}\left(\begin{array}{c}
n \\
2 s+1
\end{array}\right) \mathcal{D}_{2 s+1}(x) y^{n-2 s-1} .
$$

The first few polynomials are:

$$
\begin{aligned}
& \mathcal{D}_{0}(z)=1, \\
& \mathcal{D}_{1}(z)=x+i y, \\
& \mathcal{D}_{2}(z)=x^{2}-y^{2}+1+2 x y i \\
& \mathcal{D}_{3}(z)=x^{3}+3 x-3 x y^{2}+2+i\left(-y^{3}+3 x^{2} y+3 y\right) .
\end{aligned}
$$

In particular, for $y=0$ or $x=y=0$, we have

$$
\mathcal{D}_{n}(z)=\mathcal{D}_{n}(x), \quad \mathcal{D}_{n}(0)=\mathcal{D}_{n}
$$

\section{Some properties of the derangement polynomials with a complex variable}

In this section, we give some properties of the $\mathcal{D}_{n}(z), \mathcal{D}_{n, 1}(z), \mathcal{D}_{n, 2}(z)$.

Lemma 2.1. For any non-negative integer $n$, we have

$$
\begin{aligned}
\mathcal{D}_{n}(z) & =\sum_{k=0}^{n}(n)_{k}\left[\sum_{s=0}^{k} \frac{(x-1)^{s}}{s !}\right](i y)^{n-k}, \\
\mathcal{D}_{n, 1}(z) & =\sum_{k=0}^{n}(n)_{k}\left[\sum_{s=0}^{k} \frac{(x-1)^{s}}{s !}\right](i y)^{n-k}\left(1+(-1)^{n-k}\right), \\
\mathcal{D}_{n, 2}(z) & =\sum_{k=0}^{n}(n)_{k}\left[\sum_{s=0}^{k} \frac{(x-1)^{s}}{s !}\right](i y)^{n-k}\left(1-(-1)^{n-k}\right),
\end{aligned}
$$

where $(n)_{k}$ is the falling factorial defined by

$$
(n)_{k}=n(n-1) \cdots(n-k+1) \text { if } k \geq 1 \text { and }(n)_{0}=1 .
$$


Proof. We have

$$
\begin{aligned}
\mathcal{D}_{n}(z) & =\sum_{k=0}^{n}\left(\begin{array}{l}
n \\
k
\end{array}\right) \mathcal{D}_{k}(x)(i y)^{n-k} \\
& =\sum_{k=0}^{n} \frac{n !}{k !(n-k) !} k !\left[\sum_{s=0}^{k} \frac{(x-1)^{s}}{s !}\right](i y)^{n-k} \\
& =\sum_{k=0}^{n}(n)_{k}\left[\sum_{s=0}^{k} \frac{(x-1)^{s}}{s !}\right](i y)^{n-k} .
\end{aligned}
$$

Proposition 2.2. For any non-negative integer $n$ there holds

$$
\begin{aligned}
\mathcal{D}_{n+1}(z) & =(n+1) \mathcal{D}_{n}(z)+(z-1)^{n+1}, \\
\mathcal{D}_{n+2}(z) & =(n+1)\left[\mathcal{D}_{n+1}(z)+\mathcal{D}_{n}(z)\right]+(z-1)^{n+1}+(z-1)^{n+2}, \\
\mathcal{D}_{n}(\bar{z}) & =\overline{\mathcal{D}_{n}(z)} \\
\mathcal{D}_{n+1,1}(z) & =(n+1) \mathcal{D}_{n, 1}(z)+(z-1)^{n}+(\bar{z}-1)^{n}, \\
\mathcal{D}_{n+1,2}(z) & =(n+1) \mathcal{D}_{n, 2}(z)+(z-1)^{n}-(\bar{z}-1)^{n},
\end{aligned}
$$

where $\overline{\mathcal{D}_{n}(z)}$ is the complex conjugate of $\mathcal{D}_{n}(z)$

Proof. For (2), we have

$$
\begin{aligned}
\mathcal{D}_{n+1}(z) & =\sum_{k=0}^{n+1}(n+1)_{k}\left[\sum_{s=0}^{k} \frac{(x-1)^{s}}{s !}\right](i y)^{n+1-k} \\
& =(n+1) \sum_{k=1}^{n+1}(n)_{k-1}\left[\sum_{s=0}^{k} \frac{(x-1)^{s}}{s !}\right](i y)^{n+1-k}+(i y)^{n+1} \\
& =(n+1)\left[\sum_{k=0}^{n}(n)_{k}\left[\sum_{s=0}^{k} \frac{(x-1)^{s}}{s !}+\frac{(x-1)^{k+1}}{(k+1) !}\right](i y)^{n-k}\right]+(i y)^{n+1},
\end{aligned}
$$

then

$$
\mathcal{D}_{n+1}(z)=(n+1) \mathcal{D}_{n}(z)+(n+1)\left[\sum_{k=0}^{n}(n)_{k} \frac{(x-1)^{k+1}}{(k+1) !}(i y)^{n-k}\right]+(i y)^{n+1} .
$$

On the other hand, we have

$$
\begin{aligned}
(n+1) \sum_{k=0}^{n}(n)_{k} \frac{(x-1)^{k+1}}{(k+1) !}(i y)^{n-k}+(i y)^{n+1} & =\sum_{k=0}^{n}\left(\begin{array}{c}
n+1 \\
k+1
\end{array}\right)(x-1)^{k+1}(i y)^{n-k}+(i y)^{n+1} \\
& =\sum_{k=1}^{n+1}\left(\begin{array}{c}
n+1 \\
k
\end{array}\right)(x-1)^{k}(i y)^{n+1-k}+(i y)^{n+1} \\
& =\left[(x-1+i y)^{n+1}-(i y)^{n+1}\right]+(i y)^{n+1} \\
& =(x-1+i y)^{n+1} .
\end{aligned}
$$


Hence

$$
\mathcal{D}_{n+1}(z)=(n+1) \mathcal{D}_{n}(z)+(x-1+i y)^{n+1},
$$

or equivalently

$$
\mathcal{D}_{n+1}(z)=(n+1) \mathcal{D}_{n}(z)+(z-1)^{n+1}
$$

For (3), we have

$$
\begin{aligned}
\mathcal{D}_{n+2}(z) & =(n+2) \mathcal{D}_{n+1}(z)+(z-1)^{n+2} \\
& =(n+1) \mathcal{D}_{n+1}(z)+\mathcal{D}_{n+1}(z)+(z-1)^{n+2} \\
& =(n+1)\left[\mathcal{D}_{n+1}(z)+\mathcal{D}_{n}(z)\right]+(z-1)^{n+1}+(z-1)^{n+2} .
\end{aligned}
$$

The first few $\mathcal{D}_{n}(z)$ polynomials can be written as follows:

$$
\mathcal{D}_{0}(z)=1, \mathcal{D}_{1}(z)=z, \mathcal{D}_{2}(z)=z^{2}+1, \mathcal{D}_{3}(z)=z^{3}+3 z+2 .
$$

Note that $\mathcal{D}_{n}(z)$ is a polynomial with integer coefficients.

Proposition 2.3. Let $z_{0}$ and $z=z_{0}+h$ be two points. The function $\mathcal{D}_{n}(z)$ is holomorphic on $\mathbb{C}$ and for any non-negative integer $n$, we have

$$
\begin{aligned}
& \mathcal{D}_{n}^{\prime}(z)=n \mathcal{D}_{n-1}(z) \\
& \mathcal{D}_{n}(z)=\sum_{k=0}^{n}\left(\begin{array}{l}
n \\
k
\end{array}\right) \mathcal{D}_{n-k}\left(z_{0}\right)\left(z-z_{0}\right)^{k} .
\end{aligned}
$$

If $z_{0}=0$, we obtain

$$
\mathcal{D}_{n}(z)=\sum_{k=0}^{n}\left(\begin{array}{l}
n \\
k
\end{array}\right) \mathcal{D}_{n-k} z^{k},
$$

where $\mathcal{D}_{n}^{\prime}(z)$ is the derivative of $\mathcal{D}_{n}(z)$

Proof. For (4), we proceed by induction on $n$. Indeed, for $n=1, \mathcal{D}_{1}(z)=z$, we have

$$
\mathcal{D}_{1}^{\prime}(z)=1=\mathcal{D}_{0}(z) .
$$

For $n=2, \mathcal{D}_{2}(z)=z^{2}+1$, we have

$$
\mathcal{D}_{2}^{\prime}(z)=2 z=2 \mathcal{D}_{1}(z) .
$$

Assume for any integer $n \geq 1, \mathcal{D}_{n}^{\prime}(z)=n \mathcal{D}_{n-1}(z)$. Using the relationship (2), we get

$$
\begin{aligned}
\mathcal{D}_{n+1}^{\prime}(z) & =\left[(n+1) \mathcal{D}_{n}(z)+(z-1)^{n+1}\right]^{\prime} \\
& =(n+1) \mathcal{D}_{n}^{\prime}(z)+(n+1)(z-1)^{n} \\
& =(n+1) n \mathcal{D}_{n-1}(z)+(n+1)(z-1)^{n} \\
& =(n+1) \mathcal{D}_{n}(z) .
\end{aligned}
$$

For (5), we have $\mathcal{D}_{n}^{\prime}(z)=n \mathcal{D}_{n-1}(z)$, then $\mathcal{D}_{n}^{(2)}(z)=n(n-1) \mathcal{D}_{n-2}(z)$, and by induction the $k$-th derivative of $\mathcal{D}_{n}(z)$ is

$$
\mathcal{D}_{n}^{(k)}(z)=(n)_{k} \mathcal{D}_{n-k}(z),
$$

which gives

$$
\frac{\mathcal{D}_{n}^{(k)}(z)}{k !}=\left(\begin{array}{l}
n \\
k
\end{array}\right) \mathcal{D}_{n-k}(z) .
$$


Then the Taylor's series for $\mathcal{D}_{n}(z)$ is to be

$$
\begin{aligned}
\mathcal{D}_{n}(z) & =\sum_{k=0}^{n} \frac{\mathcal{D}_{n}^{(k)}\left(z_{0}\right)}{k !}\left(z-z_{0}\right)^{k} \\
& =\sum_{k=0}^{n}\left(\begin{array}{l}
n \\
k
\end{array}\right) \mathcal{D}_{n-k}\left(z_{0}\right)\left(z-z_{0}\right)^{k} .
\end{aligned}
$$

This completes the proof.

\section{Congruence on the derangement polynomials with a complex variable}

In this section, we use the properties of the classical umbral calculus to drive new congruences involving the derangement polynomials with a complex variable. The derangement polynomials with a complex variable are defined by

$$
\mathcal{D}_{n}(z)=\sum_{k=0}^{n}\left(\begin{array}{l}
n \\
k
\end{array}\right) \mathcal{D}_{n-k} z^{k}
$$

Let $\mathbf{D}$ be the derangement umbra defined by $\mathbf{D}^{n}=\mathcal{D}_{n}$, then we can define the generalized derangement umbra $\mathbf{D}_{\mathbf{z}}$ as follows

$$
\mathbf{D}_{\mathbf{z}}^{n}=\mathcal{D}_{n}(z)=\sum_{k=0}^{n}\left(\begin{array}{l}
n \\
k
\end{array}\right) \mathcal{D}_{n-k} z^{k}=(\mathbf{D}+z)^{n}
$$

For more information on the umbral calculus see $[1,2,4,5,10,11]$. In the remainder of this section, for any polynomials $f$ and $g$, with integer coefficients we denote by $f(z) \equiv g(z)$ to mean $f(z) \equiv g(z)\left(\bmod p \mathbb{Z}_{p}[z]\right)$ and for any numbers $a$ and $b$ by $a \equiv b$ we mean $a \equiv b(\bmod p)$.

Lemma 3.1. Let $f$ be a polynomial in $\mathbb{Z}[z]$ and $s$ be a non-negative integer, then for any prime $p \geq 3$, there holds

$$
\left(\mathbf{D}_{\mathbf{z}}^{p^{s}}+1\right) f\left(\mathbf{D}_{\mathbf{z}}\right) \equiv z^{p^{s}} f\left(\mathbf{D}_{\mathbf{z}}\right) .
$$

Proof. It suffices to take $f(z)=z^{n}$. We proceed by induction on $s$. For $s=1$ we have

$$
\begin{aligned}
\left(\mathbf{D}_{\mathbf{z}}^{p}+1\right) \mathbf{D}_{\mathbf{z}}^{n} & =\mathbf{D}_{\mathbf{z}}^{p+n}+\mathbf{D}_{\mathbf{z}}^{n} \\
& =(\mathbf{D}+z)^{p}(\mathbf{D}+z)^{n}+\mathbf{D}_{\mathbf{z}}^{n} \\
& \equiv\left(\mathbf{D}^{p}+z^{p}\right)(\mathbf{D}+z)^{n}+\mathbf{D}_{\mathbf{z}}^{n} \\
& =\mathbf{D}_{\mathbf{z}}^{n}+z^{p} \mathbf{D}_{\mathbf{z}}^{n}+\sum_{k=0}^{n}\left(\begin{array}{l}
n \\
k
\end{array}\right) \mathbf{D}^{n-k+p} z^{k}
\end{aligned}
$$

and by the known congruence $\mathcal{D}_{n+p} \equiv-\mathcal{D}_{n}$, or equivalently $\mathbf{D}^{n+p} \equiv-\mathbf{D}^{n}$, see [12]. So we obtain 


$$
\begin{aligned}
\left(\mathbf{D}_{\mathbf{z}}^{p}+1\right) \mathbf{D}_{\mathbf{z}}^{n} & \equiv \mathbf{D}_{\mathbf{z}}^{n}+z^{p} \mathbf{D}_{\mathbf{z}}^{n}-\sum_{k=0}^{n}\left(\begin{array}{l}
n \\
k
\end{array}\right) \mathbf{D}^{n-k} z^{k} \\
& =\mathbf{D}_{\mathbf{z}}^{n}+z^{p} \mathbf{D}_{\mathbf{z}}^{n}-(\mathbf{D}+z)^{n} \\
& =z^{p} \mathbf{D}_{\mathbf{z}}^{n}
\end{aligned}
$$

Assume it is true for $s \geq 1$. Then we have

$$
\begin{aligned}
& \mathbf{D}_{\mathbf{z}}^{n}\left(\mathbf{D}_{\mathbf{z}}^{p^{s+1}}+1\right)=\mathbf{D}_{\mathbf{z}}^{n}\left(\left(\mathbf{D}_{\mathbf{z}}^{p^{s}}+1-1\right)^{p}+1\right) \\
& \equiv \mathbf{D}_{\mathbf{z}}^{n}\left(\left(\mathbf{D}_{\mathbf{z}}^{p^{s}}+1\right)^{p}+(-1)^{p}+1\right) \\
& =\mathbf{D}_{\mathbf{z}}^{n}\left(\mathbf{D}_{\mathbf{z}}^{p^{s}}+1\right)^{p} \\
& =\left[\mathbf{D}_{\mathbf{z}}^{n}\left(\mathbf{D}_{\mathbf{z}}^{p^{s}}+1\right)\right]\left(\mathbf{D}_{\mathbf{z}}^{p^{s}}+1\right)^{p-1} \\
& \equiv z^{p^{s}} \mathbf{D}_{\mathbf{z}}^{n}\left(\mathbf{D}_{\mathbf{z}}^{p^{s}}+1\right)^{p-1} \\
& =z^{p^{s}}\left[\mathbf{D}_{\mathbf{z}}^{n}\left(\mathbf{D}_{\mathbf{z}}^{p^{s}}+1\right)\right]\left(\mathbf{D}_{\mathbf{z}}^{p^{s}}+1\right)^{p-2} \\
& \equiv z^{2 p^{s}} \mathbf{D}_{\mathbf{z}}^{n}\left(\mathbf{D}_{\mathbf{z}}^{p^{s}}+1\right)^{p-2} \\
& \vdots \\
& \equiv\left(z^{p^{s}}\right)^{p} \mathbf{D}_{\mathbf{z}}^{n} \\
& =z^{p^{s+1}} \mathbf{D}_{\mathbf{z}}^{n} \text {. }
\end{aligned}
$$

and the proof of the induction step is complete.

The principal result given by the following Theorem.

Theorem 3.2. For any integers $n, s \geq 1, m \geq 0$ and for any prime $p \geq 3$, there holds

$$
\mathcal{D}_{n+m p^{s}}(z) \equiv\left(z^{p^{s}}-1\right)^{m} \mathcal{D}_{n}(z)
$$

For $y=0$ or $z=0$, we obtain

$$
\begin{aligned}
\mathcal{D}_{n+m p^{s}}(x) & \equiv\left(x^{p^{s}}-1\right)^{m} \mathcal{D}_{n}(x), \\
\mathcal{D}_{n+m p^{s}} & \equiv(-1)^{m} \mathcal{D}_{n}, \\
\mathcal{D}_{n+2 p} & \equiv \mathcal{D}_{n} .
\end{aligned}
$$

Proof. For $m=1$ just take $f(z)=z^{n}$ in Lemma 3.1 and for $m>1$,we have

$$
\begin{aligned}
\mathcal{D}_{n+m p^{s}}(z) & =\mathcal{D}_{n+(m-1) p^{s}+p^{s}}(z) \\
& \equiv\left(z^{p^{s}}-1\right) \mathcal{D}_{n+(m-1) p^{s}}(z) \\
& =\left(z^{p^{s}}-1\right) \mathcal{D}_{n+(m-2) p^{s}+p^{s}}(z) \\
& \equiv\left(z^{p^{s}}-1\right)^{2} \mathcal{D}_{n+(m-2) p^{s}}(z) \\
& =\left(z^{p^{s}}-1\right)^{2} \mathcal{D}_{n+(m-3) p^{s}+p^{s}}(z) \\
& \equiv\left(z^{p^{s}}-1\right)^{3} \mathcal{D}_{n}(z) \\
& \vdots \\
& \equiv\left(z^{p^{s}}-1\right)^{m} \mathcal{D}_{n}(z) .
\end{aligned}
$$

Hence the proof is complete. 
Corollary 3.2.1. For any prime number $p \geq 3$ and any integers $s \geq 1, m_{0}, \ldots, m_{s} \in\{0, \ldots, p-1\}$, there holds

$$
\mathcal{D}_{m_{0}+m_{1} p+\cdots+m_{s} p^{s}}(z) \equiv\left(z^{p}-1\right)^{m_{1}}\left(z^{p^{2}}-1\right)^{m_{2}} \cdots\left(z^{p^{s}}-1\right)^{m_{s}} \mathcal{D}_{m_{0}}(z) .
$$

In particular, we have

$$
\begin{aligned}
& \mathcal{D}_{m_{1} p+\cdots+m_{s} p^{s}}(z) \equiv-\left(z^{p}-1\right)^{m_{1}}\left(z^{p^{2}}-1\right)^{m_{2}} \cdots\left(z^{p^{s}}-1\right)^{m_{s}} \\
& \mathcal{D}_{m_{1} p+\cdots+m_{s} p^{s}}(k) \equiv-(k-1)^{m_{1}+m_{2}+\cdots+m_{s}}
\end{aligned}
$$

\section{References}

[1] Benyattou, A., \& Mihoubi, M. (2018). Curious congruences related to the Bell polynomials, Quaest. Math., 41(3), 437-448.

[2] Benyattou, A., \& Mihoubi, M. (2019). Real-rooted polynomials via generalized Bell umbra. Notes on Number Theory and Discrete Mathematics, 25(2), 136-144.

[3] Darus, M., \& Ibrahim, R. (2010). On generalisation of polynomials in complex plane, Advances in Decision Sciences, 2010, (2010), 9 pages.

[4] Gertsch, A., \& Robert, A. M. (1996). Some congruences concerning the Bell numbers, Bull. Belg. Math. Soc. Simon Stevin, 3, 467-475.

[5] Gessel, I. M. (2003). Applications of the classical umbral calculus, Algebra Universalis, 49, 397-434.

[6] Kim, D. S., Kim, T., \& Lee, H. (2019). A note on Degenerate Euler and Bernoulli polynomials, Symmetry, 11, 1168.

[7] Kim, T., \& Kim, D. S. (2018). Some identities on derangement and degenerate derangement polynomials, Advances in Mathematical Inequalities and Applications, 265-277, Trends Math., Birkhauser/Springer, Singapore.

[8] Kim, T., Kim, D. S., Dolgy, D. V., \& Kwon, J. (2018). Some identities of derangement numbers. Proc. Jangjeon Math. Soc., 21(1), 125-141.

[9] Kim, T., Kim, D. S., Kwon, H.-I., \& Jang, L.-C. (2018). Fourier series of sums of products of $r$-derangement functions, J. Nonlinear Sci. Appl., 11(4), 575-590.

[10] Roman, S. (1984). The Umbral Calculus, Academic Press, Orlando, FL.

[11] Rota, G. C., \& Taylor, B. D. (1994). The classical umbral calculus, SIAM J. Math. Anal., $25,694-711$.

[12] Sun, Z.-W., \& Zagier, D. (2011). On a curious property of Bell numbers, Bull. Aust. Math. Soc., 84, 153-158. 